International Journal of Modern Physics E

(C) World Scientific Publishing Company

\title{
Pion-nucleon scattering within a gauged linear sigma model with parity-doubled nucleons
}

\author{
Susanna Wilms, Francesco Giacosa, and Dirk H. Rischke \\ Institut für Theoretische Physik \\ Johann Wolfgang Goethe-Universität \\ Max-von-Laue-Str. 1, D-60438 Frankfurt am Main, Germany \\ Received (received date) \\ Revised (revised date)
}

\begin{abstract}
We compute pion-nucleon scattering at tree-level within a gauged linear sigma model which contains the nucleon and its chiral partner. Such an investigation in principle allows to make definite predictions as to whether the main contribution to the nucleon mass comes from the chiral condensate or from the mixing with its chiral partner. We find that there seems to be no set of model parameters that allows for a simultaneous description of all experimentally measured scattering lengths and range parameters. This indicates the need to improve the dynamical ingredients of the model.
\end{abstract}

\section{Introduction}

Effective models which embody chiral symmetry and its spontaneous breakdown at low temperatures and densities, are widely used to understand the properties of light hadrons. Viable candidates obey a well-defined set of low-energy theorems 1, but they still differ in some crucial and interesting aspects such as the mass generation of the nucleon and the behavior at non-zero $T$ and $\mu$.

Here we concentrate on a gauged linear sigma model with $U(2)_{R} \times U(2)_{L}$ symmetry and parity-doubled nucleons. The mesonic sector involves scalar, pseudoscalar, vector and axialvector mesons; Vector Meson Dominance (VMD) automatically follows from gauging the symmetry group 23 . In the baryonic sector, besides the usual nucleon doublet field $N$, a second baryon doublet $N^{*}$ with $J^{P}=\frac{1}{2}^{-}$is included. As first discussed in Ref. 4 and extensively analyzed in Ref. 5 , in the so-called mirror assignment the nucleon fields $N$ and $N^{*}$ do not have vanishing mass in the chirally restored phase. The chiral condensate $\varphi$ further increases the nucleon masses and generates a mass splitting of $N$ and $N^{*}$, but is no longer solely responsible for generating the nucleon masses (see section 2 for details). Such a theoretical set-up has been used in Ref. $[6$ to study the properties of cold and dense nuclear matter. The experimental assignment for the partner of the nucleon is still controversial: the well-identified resonance $N^{*}(1535)$ is one candidate, but a very broad and not yet discovered resonance centered at about $1.2 \mathrm{GeV}$ has been proposed in Ref. $\underline{6}$. 
The aim of the present work is the calculation of pion-nucleon scattering at tree-level within the outlined model. This study constitutes a decisive test for its viability and can provide useful information to clarify the origin of the nucleon mass and its behavior in the chirally restored phase. Furthermore, important issues such as the dependence of the scattering lengths on the mass of the chiral partner $N^{*}$ and on the enigmatic $\sigma$ meson can be addressed.

This paper is organized as follows: in section 2 we briefly describe the details of the model, in section 3 we present and discuss the scattering amplitudes and finally, in section 4 , we summarize the present stage of our research and outline future developments.

\section{The gauged linear sigma model with parity-doubled nucleons}

The scalar and pseudoscalar fields are included in the matrix $\Phi=(\sigma+i \eta) t^{0}+$ $\left(\vec{a}_{0}+i \vec{\pi}\right) \cdot \vec{t}$ and the vector and axialvector fields are represented by the matrices $R^{\mu}=\left(\omega^{\mu}-f_{1}^{\mu}\right) t^{0}+\left(\vec{\rho}^{\mu}-\vec{a}_{1}^{\mu}\right) \cdot \vec{t}$ and $L^{\mu}=\left(\omega^{\mu}+f_{1}^{\mu}\right) t^{0}+\left(\vec{\rho}^{\mu}+\vec{a}_{1}^{\mu}\right) \cdot \vec{t}$ $\left(\vec{t}=\frac{1}{2} \vec{\tau}\right.$, where $\vec{\tau}$ are the Pauli matrices and $\left.t^{0}=\frac{1}{2} 1_{2}\right)$.

The mesonic Lagrangian, as presented in Refs. $\frac{213}{2}$, is constructed by requiring invariance under local chiral transformations; this local invariance is then broken to a global one by mass terms for the vector and axialvector mesons:

$$
\begin{aligned}
\mathcal{L}_{\mathrm{mes}}= & \operatorname{Tr}\left[\left(D_{\mu} \Phi\right)^{\dagger}\left(D^{\mu} \Phi\right)-m^{2} \Phi^{\dagger} \Phi-\lambda_{2}\left(\Phi^{\dagger} \Phi\right)^{2}\right]-\lambda_{1}\left(\operatorname{Tr}\left[\Phi^{\dagger} \Phi\right]\right)^{2} \\
& +c\left(\operatorname{det} \Phi^{\dagger}+\operatorname{det} \Phi\right)+\operatorname{Tr}\left[H\left(\Phi^{\dagger}+\Phi\right)\right] \\
& -\frac{1}{4} \operatorname{Tr}\left[\left(L^{\mu \nu}\right)^{2}+\left(R^{\mu \nu}\right)^{2}\right]+\frac{m_{\rho}^{2}}{2} \operatorname{Tr}\left[\left(L^{\mu}\right)^{2}+\left(R^{\mu}\right)^{2}\right],
\end{aligned}
$$

where $D^{\mu} \Phi=\partial^{\mu} \Phi+i g\left(\Phi R^{\mu}-L^{\mu} \Phi\right)$.

The baryon sector involves the baryon doublets $\Psi_{1}$ and $\Psi_{2}$, where $\Psi_{1}$ has positive parity and $\Psi_{2}$ negative parity. While the former transforms in the standard fashion under chiral transformations, the latter is postulated to transform according to the mirror assignment, namely:

$$
\begin{aligned}
& \Psi_{1 R} \longrightarrow U_{R} \Psi_{1 R}, \bar{\Psi}_{1 R} \longrightarrow \bar{\Psi}_{1 R} U_{R}^{\dagger}, \\
& \Psi_{2 R} \longrightarrow U_{L} \Psi_{2 R}, \bar{\Psi}_{2 R} \longrightarrow \bar{\Psi}_{2 R} U_{L}^{\dagger},
\end{aligned}
$$

and similarly for the left-handed fields. Such field transformations allow to write down the following chirally invariant Lagrangian:

$$
\begin{aligned}
\mathcal{L}_{\text {nucl }}= & \bar{\Psi}_{1 L} i \gamma_{\mu} D_{L}^{\mu} \Psi_{1 L}+\bar{\Psi}_{1 R} i \gamma_{\mu} D_{R}^{\mu} \Psi_{1 R}+\bar{\Psi}_{2 L} i \gamma_{\mu} D_{R}^{\mu} \Psi_{2 L}+\bar{\Psi}_{2 R} i \gamma_{\mu} D_{L}^{\mu} \Psi_{2 R} \\
& -\widehat{g}_{1}\left(\bar{\Psi}_{1 L} \Phi \Psi_{1 R}+\bar{\Psi}_{1 R} \Phi^{\dagger} \Psi_{1 L}\right)-\widehat{g}_{2}\left(\bar{\Psi}_{2 L} \Phi^{\dagger} \Psi_{2 R}+\bar{\Psi}_{2 R} \Phi \Psi_{2 L}\right) \\
& -m_{0}\left(\bar{\Psi}_{1 L} \Psi_{2 R}-\bar{\Psi}_{1 R} \Psi_{2 L}-\bar{\Psi}_{2 L} \Psi_{1 R}+\bar{\Psi}_{2 R} \Psi_{1 L}\right)
\end{aligned}
$$

where $D_{R}^{\mu}=\partial^{\mu}-i g R^{\mu}, D_{L}^{\mu}=\partial^{\mu}-i g L^{\mu}$.

The last term, whose strength is parameterized by $m_{0}$, plays a crucial role in the model. It is a quadratic mixing term for the nucleon fields $\Psi_{1}$ and $\Psi_{2}$. The 
physical fields $N$ and $N^{*}$, referring to the nucleon and to its chiral partner, arise by diagonalizing the Lagrangian $\mathcal{L}_{\text {nucl }}$ and are a superposition of the two fields $\Psi_{1}$ and $\Psi_{2}$ :

$$
\left(\begin{array}{c}
N \\
N^{*}
\end{array}\right)=\frac{1}{\sqrt{2 \cosh \delta}}\left(\begin{array}{cc}
e^{\delta / 2} & \gamma_{5} e^{-\delta / 2} \\
\gamma_{5} e^{-\delta / 2} & -e^{\delta / 2}
\end{array}\right)\left(\begin{array}{l}
\Psi_{1} \\
\Psi_{2}
\end{array}\right) .
$$

The parameter $\delta$, which measures the intensity of the mixing, is expressed in terms of the parameters of $\mathcal{L}_{\text {nucl }}$ as:

$$
\sinh \delta=\frac{\left(\widehat{g}_{1}+\widehat{g}_{2}\right) \varphi}{4 m_{0}},
$$

where the coupling constants $\widehat{g}_{1}$ and $\widehat{g}_{2}$ parametrize the interaction of the baryonic fields with scalar and pseudoscalar mesons and $\varphi=\langle 0|\sigma| 0\rangle=Z f_{\pi}$ is the chiral condensate. The parameter $f_{\pi}=92.4 \mathrm{MeV}$ is the pion decay constant and $Z=$ $m_{a_{1}} / m_{\rho} \approx 1.59$. The latter arises by shifting the isotriplet axialvector meson field $\vec{a}_{1}^{\mu}$ in order to avoid an unphysical mixing with the pion 7 .

The masses of the physical fields $N$ and $N^{*}$ read:

$$
m_{N, N^{*}}=\sqrt{m_{0}^{2}+\left[\frac{\left(\widehat{g}_{1}+\widehat{g}_{2}\right) \varphi}{4}\right]^{2}} \pm \frac{\left(\widehat{g}_{1}-\widehat{g}_{2}\right) \varphi}{4} .
$$

In Eq. (6) both $m_{0}$ and $\varphi$ contribute to the masses of the nucleon and its partner. Some considerations about two important limiting cases of the above listed equations are in order:

(i) When $\delta \rightarrow \infty$, corresponding to $m_{0} \rightarrow 0$, no mixing is present and $N=\Psi_{1}$, $N^{*}=\Psi_{2}$. In this case $m_{N}=\widehat{g}_{1} \varphi / 2$ and $m_{N^{*}}=\widehat{g}_{2} \varphi / 2$, thus the nucleon mass is generated solely by the chiral condensate as in the linear sigma model.

(ii) In the chirally restored phase where $\varphi \rightarrow 0$, one has mass degeneracy $m_{N}=$ $m_{N^{*}}=m_{0}$. When chiral symmetry is broken, $\varphi \neq 0$, a splitting is generated. By choosing $0<\widehat{g}_{1}<\widehat{g}_{2}$ the inequality $m_{N}<m_{N^{*}}$ is fulfilled.

In Ref. 6] a large value of the parameter $m_{0}(\sim 800 \mathrm{MeV})$ is claimed to be needed for a correct description of nuclear matter properties, thus pointing to a small contribution of the chiral condensate to the nucleon mass. Validating this claim through the evaluation of pion-nucleon scattering at zero temperature and density is subject of the present paper.

\section{The scattering amplitudes}

The general form of the Lorentz-invariant scattering amplitude can be written as 8.

$$
T_{a b}=\left[A^{(+)}+\frac{1}{2}\left(q_{1}^{\mu}+q_{2}^{\mu}\right) \gamma_{\mu} B^{(+)}\right] \delta_{a b}+\left[A^{(-)}+\frac{1}{2}\left(q_{1}^{\mu}+q_{2}^{\mu}\right) \gamma_{\mu} B^{(-)}\right] i \epsilon_{b a c} \tau_{c},
$$

where the subscripts $a$ and $b$ refer to the isospin of the initial and final states and the superscripts $(+)$ and $(-)$ denote the isospin-even and isospin-odd amplitudes, respectively. 
The pion-nucleon scattering amplitudes, evaluated from the Lagrangian $\mathcal{L}_{\text {nucl }}$ of Eq. (3) at tree-level, involve exchange of $\sigma$ and $\rho$ mesons in the $t$-channel and intermediate $N$ and $N^{*}$ states in the $s$ - and $u$-channels. At threshold, $t=0, s=$ $\left(m_{N}+m_{\pi}\right)^{2}, u=\left(m_{N}-m_{\pi}\right)^{2}$, the explicit expressions for the terms $A^{( \pm)}$and $B^{( \pm)}$ read $\left(r=m_{N^{*}} / m_{N}, y=m_{\pi} / m_{N}\right.$, threshold values are indicated by a subscript " 0 "):

$$
\begin{aligned}
A_{0}^{(+)}= & \frac{m_{N}}{Z^{4} f_{\pi}^{2}}\left\{\frac{(r-1)\left(r^{2}-1\right)}{2 \cosh ^{2} \delta} \frac{r^{2}-1-y^{2}}{\left(r^{2}-1-y^{2}\right)^{2}-4 y^{2}}\right. \\
& \left.+\left(1-\frac{r+1}{2 \cosh ^{2} \delta}\right)\left[1+\left(\frac{m_{\pi}}{m_{\sigma}}\right)^{2}\left(Z^{2}-2\right)\right]\right\}, \\
A_{0}^{(-)}= & \frac{m_{\pi}}{Z^{4} f_{\pi}^{2} \cosh ^{2} \delta} \frac{(r-1)\left(r^{2}-1\right)}{\left(r^{2}-1-y^{2}\right)^{2}-4 y^{2}}, \\
B_{0}^{(+)}= & \frac{m_{N}}{m_{\pi} Z^{4} f_{\pi}^{2}}\left[\frac{(r-1)^{2}}{\cosh ^{2} \delta} \frac{y^{2}}{\left(r^{2}-1-y^{2}\right)^{2}-4 y^{2}}-\frac{\tanh ^{2} \delta}{1-y^{2} / 4}\right], \\
B_{0}^{(-)}= & \frac{1}{2 Z^{4} f_{\pi}^{2}}\left[Z^{4}-1+\frac{\tanh ^{2} \delta}{1-y^{2} / 4}+\frac{(r-1)^{2}}{\cosh ^{2} \delta} \frac{r^{2}-1-y^{2}}{\left(r^{2}-1-y^{2}\right)^{2}-4 y^{2}}\right] .
\end{aligned}
$$

The $s$-wave and $p$-wave scattering lengths, $a_{0}^{( \pm)}$and $a_{1^{ \pm}}^{( \pm)}$, and the effective $s$-wave range of the interaction, $r_{0}^{( \pm)}$, are given by:

$$
\begin{aligned}
a_{0}^{( \pm)}= & \eta\left(A_{0}^{( \pm)}+m_{\pi} B_{0}^{( \pm)}\right), \\
a_{1^{+}}^{( \pm)}= & \frac{2}{3} \eta C_{0}^{( \pm)}, \\
a_{1^{-}}^{( \pm)}= & \frac{2}{3} \eta C_{0}^{( \pm)}-\frac{\eta}{4 m_{N}^{2}}\left[A_{0}^{( \pm)}-\left(2 m_{N}+m_{\pi}\right) B_{0}^{( \pm)}\right], \\
r_{0}^{( \pm)}= & \eta\left\{-2 C_{0}^{( \pm)}+\frac{\left(m_{N}+m_{\pi}\right)^{2}}{m_{N} m_{\pi}} D_{0}^{( \pm)}\right. \\
& \left.-\frac{1}{2 m_{N} m_{\pi}}\left[\left(1-\frac{m_{\pi}}{2 m_{N}}\right) A_{0}^{( \pm)}-\left(m_{N}+\frac{m_{\pi}^{2}}{2 m_{N}}\right) B_{0}^{( \pm)}\right]\right\},
\end{aligned}
$$

where $\eta=1 /[4 \pi(1+y)], C_{0}^{( \pm)}=\left.\frac{\partial}{\partial t}\left(A^{( \pm)}+m_{\pi} B^{( \pm)}\right)\right|_{t=0}$, and $D_{0}^{( \pm)}=\frac{\partial}{\partial s}\left(A^{( \pm)}+\right.$ $\left.m_{\pi} B^{( \pm)}\right)\left.\right|_{t=0}$. Note that the $s$-wave scattering lengths $a_{0}^{( \pm)}$correctly vanish in the chiral limit, $m_{\pi} \rightarrow 0$.

In a first study, we identify $N^{*}$ with the resonance $N^{*}(1535)$ and we fix the parameter $Z=m_{a_{1}} / m_{\rho} \approx 1.59$. The scattering lengths and the range parameter are shown as function of the mixing parameter $\delta$ in Fig. 1 for different values of the $\sigma$ mass. In the isospin-even channel, we observe a dependence on $m_{\sigma}$, which is particularly strong for $a_{0}^{(+)}$and $r_{0}^{(+)}$. However, when lowering the parameter $Z$ to $Z=\sqrt{2}$, as predicted by the KSFR relation 9 , the dependence on $m_{\sigma}$ vanishes. This can be seen directly from Eq. (8). The quantities for the isospin-odd channel are always independent of the value of the $\sigma$ mass. 

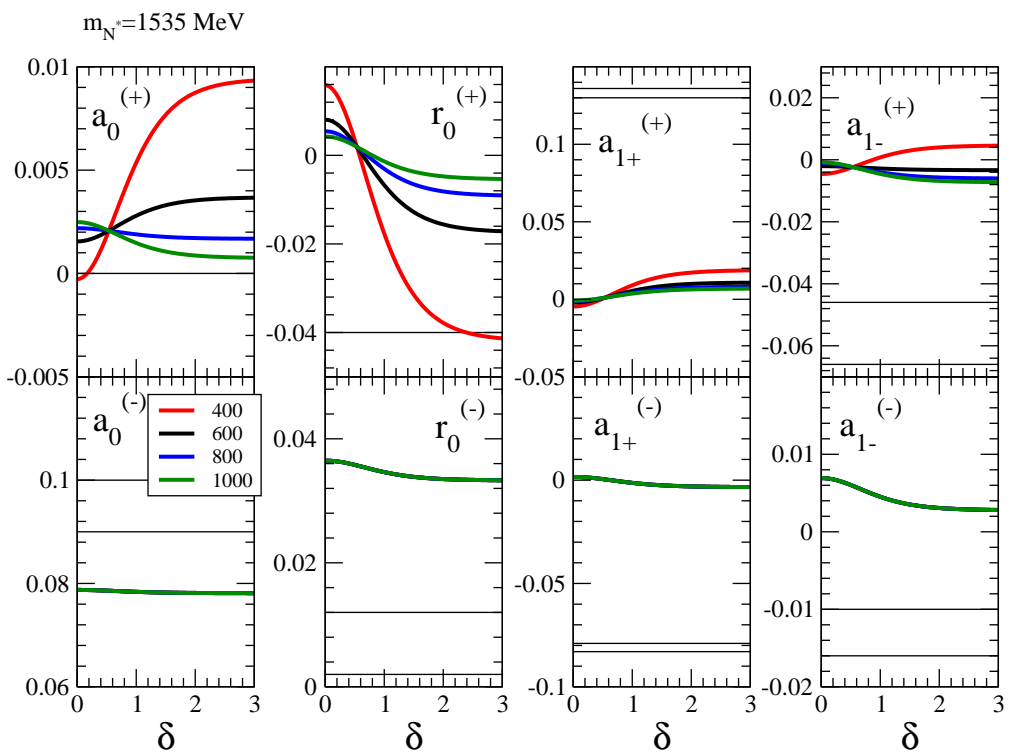

Fig. 1. Scattering lengths and range parameters as function of $\delta$, for different values of $m_{\sigma}$. The horizontal lines represent the $1 \sigma$ boundaries of the experimentally measured values.

A first, preliminary comparison of our results to the experimental data on $\pi N$ scattering lengths, as measured in Ref. 10 by precision X-ray experiments on pionic hydrogen and pionic deuterium, yields the following picture. (i) For small $m_{\sigma} \approx$ $400-600 \mathrm{MeV}$, a small value of $\delta$ is favored, in order for $a_{0}^{(+)}$to be close to the experimental data. In this case, the dominant contribution to the nucleon masses comes from $m_{0}$. (ii) For large $m_{\sigma}$, a large value for $\delta$ is required such that $a_{0}^{(+)}$ is closer to the experimental data. This points towards a dominant contribution of the chiral condensate to the nucleon mass. (iii) The effective range parameters are closer to the experimental data for large $\delta$; here, a small value for $m_{\sigma}$ is clearly favored. (iv) All $p$-wave scattering lengths come out too small in magnitude in order to compare reasonably well to the experimental data. This indicates the need to include other baryonic resonances, such as the $\Delta$.

In summary, the comparison of characteristic quantities for pion-nucleon scattering to experimental data does not unambiguously favor definite values for $m_{\sigma}$ and $\delta$. Thus, it is not (yet) possible to make a clear-cut prediction as to whether the mass of the nucleon is dominantly generated by the chiral condensate or by mixing with its chiral partner. 


\section{Summary and outlook}

In this paper we have computed the pion-nucleon scattering lengths at tree-level in the framework of a gauged linear sigma model with parity-doubled nucleons. Within the mirror assignment the mass of the nucleon originates only partially from the chiral condensate and does not vanish in the chirally restored phase. Quantitative predictions to test this scenario represent an important topic in modern hadron physics.

In a first study, we were not able to identify a definite set of parameters of our model that would simultaneously describe all scattering data. Therefore, a careful analysis of the dependence of the results on three key parameters is in order: the parameter $Z$, equal to $m_{a_{1}} / m_{\rho}$ in the mesonic sector of the Lagrangian but predicted to be $\sqrt{2}$ by the KSFR relation, the mass of the $\sigma$ meson, $m_{\sigma}$, which cannot be precisely determined due to the broadness of the corresponding resonance $f_{0}(400$ 1200), and the mass of the chiral partner of the nucleon $N^{*}$, which has not been yet unambiguously identified. Indeed, useful information toward its identification can be extracted. Then, by comparing the theoretical predictions with the experimental results we plan to determine, within our model, to which degree the nucleon mass originates from the chiral condensate and how this point is affected by the choice of the above mentioned parameters.

In a second step, we plan to extend our model by including the WeinbergTomozawa term 11 which is necessary to correctly reproduce the axial coupling constant $g_{A}$. Further applications, such as the inclusion of the $\Delta$ resonance and the study of radiative reactions, as the $\eta$-photoproduction, are also planned.

\section{Acknowledgements}

The authors thank T. Kunihiro, J. Schaffner-Bielich, S. Strüber, and D. Zschiesche for useful discussions.

\section{References}

1. U. G. Meissner, Phys. Rept. 161 (1988) 213.

2. P. Ko and S. Rudaz, Phys. Rev. D 50 (1994) 6877.

3. R. D. Pisarski, arXiv:hep-ph/9503330.

4. C. DeTar and T. Kunihiro, Phys. Rev. D 39 (1989) 2805.

5. D. Jido, M. Oka, and A. Hosaka, Prog. Theor. Phys. 106 (2001) 873 arXiv:hep-ph/0110005.

6. D. Zschiesche, L. Tolos, J. Schaffner-Bielich, and R. D. Pisarski, arXiv:nucl-th/0608044

7. S. Gasiorowicz and D. A. Geffen, Rev. Mod. Phys. 41 (1969) 531.

8. T. Matsui and B. D. Serot, Annals Phys. 144 (1982) 107.

9. K. Kawarabayashi and M. Suzuki, Phys. Rev. Lett. 16 (1966) 255.

10. H. C. Schroder et al., Eur. Phys. J. C 21 (2001) 473.

11. S. Weinberg, Phys. Rev. Lett. 17 (1966) 616. Y. Tomozawa, Nuovo Cim. 46A (1966) 707 . 